\title{
Valoración de la geodiversidad. Validación metodológica en escalas detalladas ${ }^{1}$
}

\author{
David Serrano Giné2
}

\begin{abstract}
RESUMEN
Los estudios de geodiversidad todavía son poco frecuentes, aún no disponen de un cuerpo teórico y metodológico suficientemente desarrollado y han sido aplicados principalmente a pequeña escala. El propósito de este artículo es desarrollar y aplicar un procedimiento de valoración de la geodiversidad que pueda realizarse en unidades de dimensión reducida y tener validez a escala detallada. Para ello se propone un método que parte de experiencias previas, se centra en un proceso de ensayo y error sustentado por un exhaustivo trabajo de campo y se beneficia del cálculo y representación gráfica ofrecido por los sistemas de información geográfica. La validación se efectúa en Muntanyes d'Ordal (Barcelona, España). Los resultados obtenidos indican un predominio de las superficies con valoración media $(37,34 \%)$ y alta $(36,24 \%)$. Gracias al trabajo de campo se demuestra la validez del método en ámbitos escalares detallados. La investigación presta particular atención al acabado cartográfico.
\end{abstract}

Palabras clave: Geodiversidad, geomorfología, paisaje, Muntanyes d’Ordal.

\begin{abstract}
Geodiversity studies are still rare, do not have a sufficiently developed theoretical or methodological body, and are primarily applied at small scales. The purpose of this paper is to develop and implement a process for assessing geodiversity at detailed scales. To this end, a method based on previous experiences, focused on a trial and error method, supported by an extensive field-work and using geographic information systems is proposed. Validation was performed in Muntanyes d'Ordal (Barcelona, Spain). The results show a predominance of surfaces with medium $(37.34 \%)$ and high values $(36.24 \%)$. Thanks to fieldwork, the robustness of the method was demonstrated at a detailed scale. Special attention is paid to mapping aspects.
\end{abstract}

Key words: Geodiversity, geomorphology, landscape, Muntanyes d'Ordal.

1 Durante el redactado de este artículo se ha contado con el apoyo institucional del Servei de Gestió i Evolució del Paisatge de la Universidad de Barcelona. La información aquí expuesta es fruto de una investigación de mayor alcance desarrollada bajo dirección del Dr. Antonio Gómez Ortiz. Artículo recibido el 26 de febrero de 2013, aceptado el 7 de noviembre de 2013 y corregido el 23 de junio de 2014.

2 Departamento de Geografía, Universidad Rovira i Virgili (España). E-mail: david.serrano@urv.cat 
De un tiempo a esta parte los estudios que evalúan la diversidad del medio natural están tomando un protagonismo creciente. Resulta habitual percibir estos trabajos desde un posicionamiento holístico, si bien rápidamente se diferencian aquellos aspectos relacionados con la diversidad de elementos biológicos, o biodiversidad, y aquellos otros relacionados con la diversidad de elementos abióticos, o geodiversidad. Existen, aun, otros posicionamientos centrados en la diversidad de elementos geográficos, o de la superficie terrestre, que también reciben el nombre de geodiversidad. Esta convivencia de significados y acepciones genera cierto grado de confusión, así como dificultad a la hora de desarrollar metodologías de trabajo. La idea de biodiversidad se encuentra ampliamente difundida y goza de una cómoda aceptación, tanto por parte de la sociedad civil como en ámbitos académicos y científicos. Por el contrario la noción de geodiversidad -en su acepción de diversidad abiótica- se halla mucho más restringida, no disfruta de tanta popularidad y connota una ambigüedad claramente manifiesta. Finalmente la geodiversidad -en su acepción de diversidad territorialse asimila a la suma de ambas distinciones, a la que cabe incorporar la diversidad de elementos antrópicos. En las líneas que siguen nos centraremos en la geodiversidad entendida como diversidad de elementos abióticos, puntualizaremos de qué manera entendemos este concepto y desarrollaremos una metodología para valorarla, tomando como ámbito de referencia las escalas grandes de trabajo.

Si bien es posible encontrar un elevado número de definiciones de biodiversidad (Gaston \& Spicer, 2004), existe bastante unanimidad a la hora de reconocerlas y confrontarlas. Una de las definiciones más aceptadas es la propuesta en el artículo segundo del Convenio sobre Diversidad Biológica, promulgado en el seno de la Conferencia sobre el Medio Ambiente y el Desarrollo, de 1992, donde por diversidad biológica se entiende "la variabilidad de organismos vivos de cualquier fuente, incluidos, entre otras cosas, los ecosistemas terrestres y marinos y otros ecosistemas acuáticos y los complejos ecológicos de los que forman parte; comprende la diversidad dentro de cada especie, entre las especies y de los ecosistemas" (ONU, 1992). Esta definición ha sido laxamente trabajada, tanto desde planteamientos teóricos como aplicados y políticos (DeLong, 1996; Primack y Ros, 2002; Comisión Europea, 2006). La idea de biodiversidad mantiene estrechos vínculos con la gestión ambiental, la valoración de patrimonio natural y el establecimiento y manejo de espacios naturales protegidos (Mallarach, 2008).

El concepto de geodiversidad, por el contrario, no se halla tan desarrollado, carece de una definición unívoca ampliamente aceptada y adolece de poca difusión en el léxico e imaginario colectivo. De esta manera por geodiversidad se entienden concepciones claramente acotadas, próximas a "diversidad de geologías"; otras más convergentes, que reúnen aspectos topográficos, litológicos, geomorfológicos y estructurales; y aun otras que incluyen la totalidad de elementos abióticos del medio: "geodiversity refers to a set of interfingering spheres: the atmosphere, lithosphere, morphosphere, pedosphere, hydrosphere and biosphere" (Kozlowski, 2004: 834) o, incluso, de la superficie terrestre. La variedad de conceptualizaciones y planteamientos es notoria, hecho que ha conducido a un cierto número de experiencias, tanto teóricas como aplicadas (Gray, 2004; Haffey, 2008). Por todo ello se conoce que el concepto de geodiversidad interdigita con otros campos afines, como los relacionados con valores patrimoniales y de conservación. Si bien es habitual entender su participación desde posiciones meramente escénicas, cuando no estéticas (Gronggrijp, 1999; Fernández y Pradas, 2002).

La diversidad natural, sea como fuere, abraza tanto aquellos aspectos de orden biótico como aquellos otros de carácter abiótico; es por ello que propuestas globalizadoras como diversidad biofísica o diversidad del medio natural parecen ser totalmente acertadas. La idea de diversidad territorial, por otra parte, agrega a estas dos esferas la concerniente al ser humano y sus actividades. Conviene, no obstante, considerar que las definiciones y metodologías relacionadas con el estudio de la biodiversidad no se corresponden completamente con aquellas otras ligadas a la geodiversidad, si bien se admite el vínculo evidente entre ambos conceptos. Autores como Lars Erikstad (1999), Carl Johansson et al. (1999) o Murray Gray (2004), entre otros, reflexionan a propósito de esta relación, asegurando que la valorización y la 
conservación de la biodiversidad derivan de la valorización y conservación de la geodiversidad, por ser esta la base y el soporte de organismos y ecosistemas:

"biodiversity depends essentially on geodiversity - on the geological environment with its variations. For instance, some plants need certain minerals and elements; certain plant societies are rooted in specific substrata, and some animals are adapted to sand dunes, others to river banks" (Johansson et al., 1999: 2).

En las líneas que siguen se desarrolla y aplica un método de valoración de la geodiversidad, en una zona bien conocida por nosotros, donde aún no se han realizado estudios de estas características. El método que se implementa se concibe de forma holística para escalas grandes de trabajo, y nace de aplicar y corregir experiencias similares que, por haberse desarrollado desde otros planteamientos $u$ otros contextos escalares, no propician resultados satisfactorios en estudios de detalle.

Con el propósito arriba indicado, se reúnen distintas experiencias previas que permiten reflexionar sobre el concepto de geodiversidad, valorar los métodos empleados al respecto $y$, finalmente, derivar una propuesta ajustada al área de estudio, la cual verificamos a través de un exhaustivo trabajo de campo. Tras la presentación del área de estudio y el desarrollo de la metodología empleada, se exponen los resultados obtenidos. Este último punto cede paso a la discusión de los mismos, en un fructífero apartado donde se aplican y comparan otros métodos de trabajo aplicados con éxito en distintos ámbitos territoriales pero con resultados poco acertados en nuestra área de trabajo. Finalmente se señala la oportunidad de replicar el procedimiento propuesto en planteamientos y ámbitos escalares similares, como medida para validar su bondad y facilitar su aplicación desde concepciones holísticas y ámbitos territoriales detallados.

\section{La geodiversidad}

Se conoce un buen número de definiciones de geodiversidad, no todas coincidentes y no todas elaboradas desde un mismo planteamiento. Algunas propuestas asimilan la geodiversidad a diversidad de elementos geográficos o, por lo menos, a diversidad de elementos de la superficie terrestre. Algunas escuelas clásicas, como la Geografía Cultural de Federico Alberto Daus, se sitúan en esta perspectiva. Otras propuestas precisan más su campo de trabajo, y asimilan el prefijo geo a los elementos abióticos de la superficie terrestre. Estos planteamientos se definen por negación respecto la biodiversidad, la cual se centra en elementos bióticos, al tiempo que permiten un distanciamiento de aquellas otras posiciones que focalizan en cuestiones de carácter antrópico. Este último enfoque, geodiversidad como diversidad de elementos abióticos, ha generado de manera reciente una pródiga línea de trabajo desde donde, bajo distintas consideraciones y perspectivas, se busca identificar y valorar aquellas porciones de la superficie terrestre con mayor número y diversidad de elementos y dinámicas de predominio abiótico.

En este sentido, Luis Miguel Nieto (2001) recoge las propuestas más significativas y las agrupa en tres grandes conjuntos, según si atienden a diversidad geológica, estructuras, materiales y procesos geológicos o diversidad natural y ordenación y gestión del territorio. El trabajo de Nieto también hace referencia a distintos documentos con valor normativo, así como a experiencias concretas realizadas desde diferentes concepciones, hecho que refuerza la dispersión de planteamientos y métodos de trabajo existente.

Luis Carcavilla, Jerónimo López y Juan José Durán (2007) reflexionan sobre el concepto de geodiversidad, señalando las relaciones que mantiene con el patrimonio geológico, la biodiversidad y el paisaje. Los autores conciben la geodiversidad de manera restrictiva, asimilándola estrictamente a diversidad geológica e indicando su estudio a partir de rasgos geológicos (como los afloramientos, las formas del terreno o los elementos unitarios), sus límites y los procesos que los han generado; con todo admiten vínculos con otros elementos del territorio, caso de la geografía, el paisaje, el clima, la cultura o la economía. De esta manera proponen una definición de geodiversidad centrada, precisamente, en la idea de diversidad geológica: "la variabilidad de litologías e intervalos cronoestratigráficos presentes en el registro 
geológico de un determinado territorio, en relación con su abundancia, distribución, frecuencia y, si se analiza su relación con el patrimonio geológico, importancia geológica" (Carcavilla et al., 2007: 152).

Por otra parte, Enrique Serrano y Purificación Ruiz (2007a) señalan de manera clara la existencia de distintos planteamientos de trabajo en función del grado de restricción con el que se concibe este término. Indican la existencia de consideraciones excluyentes, que por geodiversidad entienden únicamente diversidad geológica, así como planteamientos más abiertos, que integran en un solo concepto la totalidad de elementos físicos del medio natural. En este sentido proponen el estudio de la geodiversidad a partir de cinco elementos (topografía, geología, geomorfología, hidrología y suelos), desglosables en distintos subelementos y desde cuatro jerarquías escalares, según si la geodiversidad atiende a partículas, elementos, lugares o paisajes. De la misma manera plantean una definición con marcada voluntad holística, que no solo contempla elementos y procesos sino también interrelaciones y rangos escalares incluyendo, asimismo, la participación del ser humano:

"Thus, in acknowledgement of the elements targeted by the concept and, in particular, in recognition of the integrative character of the biotic elements of a natural system, it is put forward here that geodiversity be defined from a theoretical point of view as the variability of abiotic nature, including lithological, tectonic, geomorphological, soil, hydrological, topographical elements and physical processes on the land surface and in the seas and oceans, together with systems generated by natural, endogenous and exogenous, and human processes, which cover the diversity of particles, elements and places" (Serrano \& Ruiz, 2007b:143-144).

Los lugares con una geodiversidad interesante han sido estudiados y clasificados bajo distintos parámetros en función de los valores que albergan, los procesos que se desarrollan y el alcance espacial de los mismos (Reynard, 2004a; Reynard y Panizza, 2005; Calvet y Giusti, 2009), hecho que ha llevado a vincular la geodiversidad con los espacios protegidos. Autores como Juan José
González y Enrique Serrano (2008) indican que numerosos espacios protegidos acogen importantes elementos de geodiversidad, a pesar de que estos no siempre aparecen recogidos en los motivos de su declaración y por bien que esta acostumbre a realizarse bajo justificaciones más relacionadas con lo biótico que con lo abiótico. Aparte cabe contar la preponderancia de elementos y criterios de tipo biológico, tanto en cuestiones relacionadas con la gestión y el mantenimiento de espacios protegidos como en su evaluación (Mallarach, 2008). Iniciativas como el trabajo sobre geosites de la IUGS (Wimbledon et al., 2000) o el Manifiesto Europeo para el Patrimonio Terrestre y la Geodiversidad, de 2004, pretenden corregir esta desviación.

Mario Panizza y Sandra Piacente (2003) han trabajado la valorización de espacios protegidos por su geodiversidad. Estos autores destacan la ligazón entre geodiversidad y patrimonio cultural y, a través de él, con aqueIlos aspectos de tipo social que habitualmente no se relacionan con temáticas abióticas. De hecho la consideración de estos lugares puede realizarse atendiendo a tres aspectos, a saber: la investigación científica (incluyendo la identificación, el censo, la evaluación y la protección de elementos y lugares), la integración cultural (en la medida en que estos lugares conforman un paisaje sentido como propio por la sociedad) y la inspiración artística (atendiendo a la sugestión que puedan despertar en la elaboración de obras literarias, pictóricas y, en un sentido amplio, artísticas) (Panizza \& Piacente, 2002). Como resulta lógico inferir, de esta concepción deriva la riqueza patrimonial, o geopatrimonial, de aquellos lugares con valores geomorfológicos originales (Castro et al., 2012). Todo ello lleva a hablar de la geodiversidad como recurso ambiental, social y económico, por ejemplo mediante actividades como el turismo (Voth, 2004; Pralong, 2005; Naharaoui, 2011). Esta complementa otras valoraciones territoriales, ya centradas en aspectos bióticos, ya en otros de tipo cultural o social, y se sitúa en la línea de estudios como los propuestos por el Montpellier Panel, que se aproximan a territorios y sociedades atendiendo a las distintas dimensiones de su complejidad.

Como resulta fácil imaginar se han desarrollado experiencias en distintos ámbitos 
escalares, si bien aquellos poco detallados parecen resultar especialmente habituales. En cualquier caso, el papel de los elementos abióticos en el paisaje y, particularmente, la de aquellos relacionados con la geodiversidad, se ha puesto de manifiesto en numerosas ocasiones (Chevalier, 1928; Reynard, 2004b, Castro y Brignardello, 2005; Reynard, 2005; Gómez Ortiz et al., 2010; Serrano Giné, 2012). Esta participación se debe fundamentalmente al peso que poseen estos elementos en la configuración del paisaje, a causa de su rol estructurante y su predominio visual, que los incluyen e individualizan, al mismo tiempo, en el conjunto donde se encuentran: "Mais le relief peut-il se confondre avec le paysage? Si l'on considère que pour qu'il y ait paysage, il faut que s'établisse une relation entre une portion de l'espace géographique et un observateur et que cette relation introduit une série de filtres perceptifs entre l'espace géographique et ce que nous appelons le paysage. Il participe bien sûr à la création de paysages, mais il n'est pas le paysage. $C^{\prime}$ est ce que nous avons appelé le paysage géomorphologique, que l'on pourrait définir comme " une portion du relief terrestre, vue, perçue (et parfois exploitée) par l'Homme»" (Reynard, 2005: 14). De esta manera a través del paisaje la geodiversidad adquiere una dimensión netamente patrimonial, tal como se evidencia, por ejemplo, en el caso de las actividades mineras (Carvajal y González, 2002).

\section{Evaluación de la geodiversidad}

Las evaluaciones de diversidad natural se basan en la selección, clasificación y ponderación de los distintos elementos que componen el medio. Ello se realiza a través de criterios, métodos y modelos, centrados en los valores y calidades de dichos elementos, sean o no cuantificables. Este hecho comporta obstáculos de distinto orden que, según Josep Maria Mallarach (1999), pueden ser de tipo ideológico, procedimental o metodológico. Con todo, no conviene olvidar que la consideración que se disponga a estos elementos y criterios de evaluación, sea cual fuere, deriva de la importancia que la sociedad les atribuya y, por tanto, siempre puede resultar variable y discutible.

Wimbledon et al. (2000) realizan la selección de lugares de interés geológico mediante criterios de representatividad, singularidad, aptitud o idoneidad, estudio multidisciplinar global, disponibilidad y potencialidad, complejidad y (geo)diversidad. Viola Bruschi (2007: 82) señala que la evaluación de lugares de interés geológico se efectúa mediante la identificación de criterios de calidad (valor intrínseco, potencial de uso y grado de amenaza), la especificación de las características y parámetros que permiten representarlos, el establecimiento de distintas escalas de valor para cada parámetro y la integración de los mismos para generar un "modelo de calidad". Un planteamiento distinto fija una mayor atención en los aspectos culturales que se vinculan a estos lugares, particularmente mediante la interrelación entre formas del relieve y actuaciones humanas (Panizza \& Piacente, 2003). Serrano Cañadas y González Trueba (2005) demuestran la utilidad del cuaderno de campo y las fichas normalizadas para recoger información de lugares de interés geomorfológico; los autores proponen una evaluación a partir de cinco parámetros de tipo cultural y nueve de uso y gestión, que son puntuados, respectivamente, en dos y tres escalas cuantitativas. Murray Gray (2004) señala que la valoración de la geodiversidad puede desglosarse atendiendo a cinco apartados, a saber valores de tipo cultural, estético, económico, funcional y de investigación y educación. En el ámbito del paisaje Reynard (2004a) apunta que la valoración puede dirigirse hacia aspectos de tipo geocientífico, geoecológico, estético, cultural, religioso e histórico y económico. Desde un posicionamiento mucho más amplio Mallarach (1999) especifica que los criterios a seguir pueden ser de carácter ecológico, de planificación y gestión y cultural, subdividiéndose cada uno de ellos en distintos subapartados.

Las metodologías específicas para valorar la geodiversidad no son especialmente abundantes. La propuesta de Kozlowski (2004) para Polonia considera cuatro elementos (desglosados en trece subelementos) en cinco categorías posibles (que varían de "muy alto" a "muy bajo"), utilizando como base territorial ocho mapas a escala 1:750.000 donde se representa la geodiversidad de la estructura geológica, de la superficie terrestre, de los suelos, de las aguas superficiales, de las aguas subterráneas, de las aguas minerales y terapéuticas, de las aguas termales y de la estructura del paisaje en clave de conservación 
de la geodiversidad. Nieto (2001) expone una iniciativa que considera el número, variedad, naturaleza y extensión de puntos de interés geológico (PIG) y puntos de valor intrínseco (PIV) por unidad de superficie, señalando específicamente la subjetividad con la que se escogen los PIG y PIV objeto de análisis, así como la lógica variación de resultados que existe al comparar áreas de distinta superficie. Por otra parte, Carcavilla et al. (2007) proponen la valoración de la geodiversidad a partir del análisis de la diversidad, frecuencia y distribución de entidades geológicas, con el fin de cuantificarlas y facilitar su comparación. Ello comporta acotar la muestra a analizar y la definición de clases, a partir del número y variabilidad de elementos considerados, y teniendo muy presente la importancia de la escala de trabajo. Una vez establecidas dichas clases se propone el uso de algoritmos que faciliten su análisis, atendiendo a parámetros de abundancia, frecuencia, distribución y gradientes de geodiversidad. Finalmente, Serrano y Ruiz (2007a) aplican un sencillo algoritmo que relaciona la variedad de elementos físicos con la rugosidad y la dimensión y desarrollan su aplicación a pequeña escala, utilizando como espacio de referencia unidades geomorfológicas previamente delimitadas. Los mismos autores señalan la importancia de la rugosidad como variable para colegir información sobre topografía y variaciones microclimáticas y topoclimáticas, así como la poca adecuación del método a unidades de pequeño tamaño.

\section{Metodología}

La experiencia en metodologías para valorar la geodiversidad es limitada. Las aplicaciones a casos concretos son escasas y, si se contrastan entre sí, no resultan proclives a la realización de comparaciones.

Autores como Panizza y Piacente (2003), Gray (2004), Reynard (2004) y Pralong (2005) han realizado importantes apreciaciones a cerca de la geodiversidad y su valoración. Otros autores, como Serrano y Ruiz (2007a, 2007b) o Carcavilla et al. (2007) han especificado estos planteamientos en metodologías concretas, que validan en ámbitos escalares pequeños. A partir del conocimiento del área de estudio, y gracias a las aportaciones de estos distintos autores, se diseña un procedimiento de trabajo que evalúa la geodiversidad. Este procedimiento atiende a la variedad y a la abundancia de elementos físicos, a la frecuencia, distribución y superficie de las unidades de análisis y a la pendiente y rugosidad del territorio estudiado, y presenta la particularidad de desarrollarse en un ámbito escalar de sumo detalle.

La consideración de los distintos parámetros se realiza tomando como ámbito de referencia unidades homogéneas de relieve. Estas delimitaciones se han realizado bajo criterios de homogeneidad topográfica, geológica y geomorfológica, pero sin confundirlas con unidades litológicas o geomorfológicas stricto sensu. Dichas delimitaciones, pues, deben entenderse como un catálogo de tipos de relieve, en el sentido amplio de dicho concepto. Su objetivo es delimitar áreas con características similares desde el punto de vista de la topografía, la morfoestructura, la morfodinámica y, por extensión, el relieve. Tienen un eminente carácter sintético, ya que definen y delimitan espacios homogéneos desde una perspectiva abiótica. Las unidades de relieve establecidas tienen validez a escala 1:20.000 y se concretizan en 408 delimitaciones reunidas en 36 unidades (Serrano Giné, 2012).

El procedimiento seguido para valorar la geodiversidad se estructura en la delimitación de las unidades de relieve, la recopilación de los elementos físicos contenidos en ellas y su posterior correlación, a través de los siguientes seis pasos:

a) Análisis del área de estudio mediante trabajo de gabinete y exhaustivo trabajo de campo

b) Establecimiento de unidades de relieve, delimitadas bajo criterios de homogeneidad topográfica, geológica y geomorfológica, con validez a 1:20.000

c) Inventario de los elementos abióticos contenidos en cada unidad delimitada

d) Evaluación de la geodiversidad mediante el siguiente cálculo:

$$
\mathrm{Gd}=\mathrm{Eg} * \mathrm{Rq}^{*} \mathrm{Sn} * \mathrm{Un} * \mathrm{P} * \mathrm{Rg}
$$

e) Cartografiado de los resultados obtenidos

f) Verificación con la realidad 
El valor Eg del algoritmo aplicado nace del recuento de los elementos físicos existentes en la unidad (Cuadro $N^{\circ} 1$ ). Contabiliza el número de elementos diferentes, contemplando tanto elementos relacionados con la litología, morfoestructuras, procesos y microformas, como elementos de carácter hidrológico o edáfico. Sin llegar a la exhaustividad de Juan José Durán (1998), los elementos observados prestan mayor atención a la geología física que a la geografía física. Conviene tener presente que algunos de estos elementos ya aparecen implícitos en la unidad de relieve donde se observan por ser, precisamente, motivadores de su definición. La abundancia de elementos contabilizados se expresa por el parámetro $\mathrm{Rq}$, que codifica la repetición de observaciones en tres categorías, donde 1 equivale a poco abundante, 2 a abundante $y$ 3 a muy abundante.

Cuadro $\mathrm{N}^{\circ} 1$

Elementos físicos existentes en cada unidad de relieve

\begin{tabular}{|c|c|c|c|c|}
\hline \multicolumn{5}{|c|}{ Tectónica y estructura } \\
\hline \multirow[t]{2}{*}{ Tectónica } & Orogenia hercínica & $\begin{array}{l}\text { Estructuras } \\
\text { antiguas }\end{array}$ & & \\
\hline & Orogenia alpina & $\begin{array}{l}\text { Estructuras de } \\
\text { cobertera }\end{array}$ & & \\
\hline \multirow[t]{4}{*}{ Estructuras } & Fallas paleozoicas & & & \\
\hline & $\begin{array}{l}\text { Fallas alpinas y } \\
\text { cabalgamientos }\end{array}$ & & & \\
\hline & Cuestas & Cobertera triásica & & \\
\hline & & $\begin{array}{l}\text { Cobertera } \\
\text { cretácica }\end{array}$ & & \\
\hline \multicolumn{5}{|c|}{ Elementos litoestratigráficos } \\
\hline Cronología y facies & & & \multicolumn{2}{|l|}{ Litología Geoforma } \\
\hline \multirow[t]{3}{*}{ Paleozoico } & Cambroordoviciano & & Esquistos & \\
\hline & & & Diabases & \\
\hline & & & Diques cuarcíticos & \\
\hline \multirow[t]{11}{*}{ Mesozoico } & \multirow[t]{7}{*}{ Triásico } & \multirow[t]{3}{*}{ Buntsandstein } & \multirow{3}{*}{$\begin{array}{l}\text { Conglomerados, } \\
\text { areniscas, lutitas }\end{array}$} & Eólicas \\
\hline & & & & Turriculares \\
\hline & & & & Minería \\
\hline & & \multirow[t]{3}{*}{ Muschelkalk } & \multirow{3}{*}{$\begin{array}{l}\text { Dolomías, calizas, } \\
\text { margas, yesos }\end{array}$} & \\
\hline & & & & Cársticas \\
\hline & & & & $\begin{array}{l}\text { Afloramientos } \\
\text { rocosos }\end{array}$ \\
\hline & & Keuper & Arcillas, evaporitas & Minería \\
\hline & Jurásico & & Calizas. dolomías & Cársticas \\
\hline & \multirow[t]{3}{*}{ Cretácico } & & \multirow[t]{3}{*}{ Calizas, dolomías } & Cársticas \\
\hline & & & & $\begin{array}{l}\text { Afloramientos } \\
\text { rocosos }\end{array}$ \\
\hline & & & & Minería \\
\hline
\end{tabular}


Continuación Cuadro $N^{\circ} 1$

\begin{tabular}{|c|c|c|c|}
\hline \multicolumn{4}{|c|}{ Tectónica y estructura } \\
\hline \multirow[t]{6}{*}{ Cuaternario } & \multirow[t]{6}{*}{ Pleistoceno } & \multirow[t]{2}{*}{ Aluviones } & $\begin{array}{l}\text { Abanicos } \\
\text { aluviales }\end{array}$ \\
\hline & & & $\begin{array}{l}\text { Terrazas } \\
\text { fluviales }\end{array}$ \\
\hline & & Coluviones & $\begin{array}{l}\text { Costras de } \\
\text { caliche }\end{array}$ \\
\hline & & \multirow[t]{2}{*}{ Aluviones } & $\begin{array}{l}\text { Abanicos } \\
\text { aluviales }\end{array}$ \\
\hline & & & $\begin{array}{l}\text { Terrazas } \\
\text { fluviales } \\
\end{array}$ \\
\hline & & Coluviones & Deslizamientos \\
\hline
\end{tabular}

Fuente: Elaboración propia.

La participación de cada unidad en el conjunto se contempla mediante el valor S y el valor $U$. El primero expresa el porcentaje en superficie (ha) de cada unidad respecto el conjunto de unidades, mientras que el segundo indica la participación relativa en número de delimitaciones de cada unidad respecto a las unidades de un mismo grupo (Serrano Giné, 2012). De esta manera se observan las dos posibles frecuencias de aparición, según si considera a la superficie relativa o a la repetición de ellas, siempre contemplando como valor de referencia el total de unidades delimitadas. Con el objetivo de valorar positivamente aquellas unidades más escasas (tanto en número como en extensión) se ha aplicado el valor inverso, que se ha obtenido mediante la formulación [100-S] o [100-U]. El resultado es la reducción equivalente, expresada bajo la forma Sn y Un.

El valor $\mathrm{P}$ corresponde a la pendiente media de la delimitación, expresada en grados. Por otra parte, el valor $\mathrm{Rg}$ atiende a la rugosidad media, entendida como magnitud de cambio espacial del relieve. Ambos valores, pendiente y rugosidad, han sido codificados según la propuesta de Serrano y Ruiz (2007a, 2007b) (Cuadro No2).

Los valores obtenidos tras la aplicación del algoritmo han sido ajustados a una clasificación de cuatro categorías, utilizando al efecto el método de clasificación de cortes naturales, o de Jenks. Este procedimiento ha sido escogido por permitir realizar agrupamientos de valores similares en clases homogéneas, atendiendo a la manera como se distribuyen los datos a lo largo del rango de la variable. Las clases resultantes han sido recodificadas en una leyenda nominal que distingue cuatro categorías: bajo, medio, alto y muy alto, según los valores numéricos calculados.

Finalmente cabe indicar que para la aplicación del logaritmo propuesto, el cálculo de la pendiente, la rugosidad y el cartografiado de los valores resultantes, ha sido de gran utilidad el uso de un sistema de informa-

Cuadro $\mathrm{N}^{\circ} 2$

Codificación empleada para valorar la pendiente y la rugosidad del relieve

\begin{tabular}{|l|r|r|r|r|r|}
\hline Pendiente $\left(^{\circ}\right) /$ Rugosidad & $0-5$ & $6-15$ & $16-25$ & $26-50$ & $>50$ \\
\hline Codificación & 1 & 2 & 3 & 4 & 5 \\
\hline
\end{tabular}

Fuente: Serrano y Ruiz, 2007a. 
ción geográfica. Por otra parte es importante señalar que los datos colectados han sido obtenidos mediante un exhaustivo trabajo de campo, y que la comprobación de resultados se ha servido, también, del conocimiento directo de la realidad.

\section{Validación metodológica}

La validación metodológica se desarrolla en Muntanyes d'Ordal, un espacio de baja montaña repartido en poco más de 15.000 ha, a escasos kilómetros de la ciudad de Barcelona (Figura $N^{\circ} 1$ ). El sector se caracteriza por la compartimentación del relieve, que se traduce en una sucesión de valles, sierras y rellanos en un contexto claramente montuoso. Las alturas máximas del sector son modestas (Puig d'Agulles, 653,1 m) pero la fragmentación y rugosidad resultan notables, e influencian claramente la vegetación y las actividades humanas. La ocupación del suelo se estructura por formaciones arbóreas y arbustivas de tipo mediterráneo, algunos cultivos de secano de distribución irregular y un rosario de urbanizaciones residenciales de baja densidad (Paül \& Serrano, 2005).

Este sector debe entenderse como un área particularmente sensible desde el punto de vista ambiental. Por una parte ello se explica por su proximidad a la ciudad de Barcelona y su inclusión en un contexto periurbano; por otra parte por la fragilidad propia de los relieves montañosos que, a pesar de ser modestos, son una constante en Muntanyes d'Ordal. Por todo ello y por la riqueza del patrimonio ambiental y cultural, este sector se encuentra

Figura $\mathrm{N}^{\circ} 1$

Localización del área de estudio

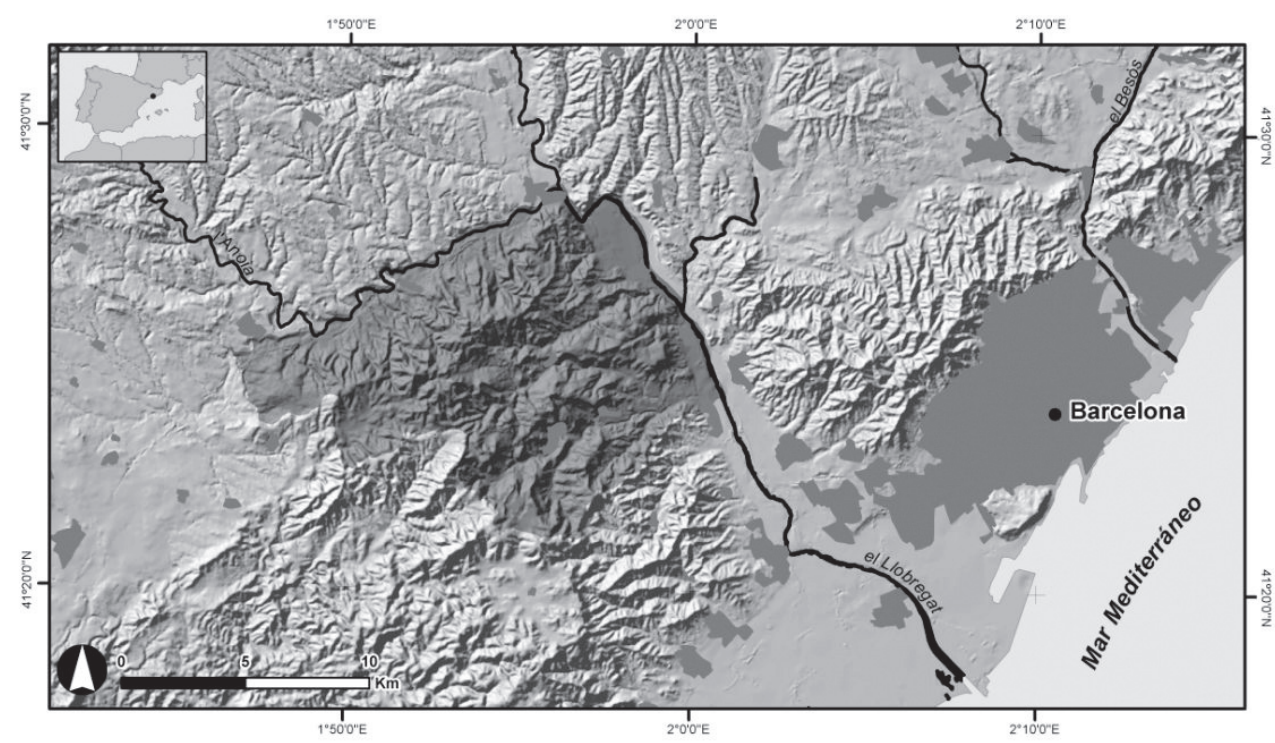

Fuente: Elaboración propia.

parcialmente protegido por la Unión Europea por el Lugar de Interés Comunitario "Serres del Litoral Central", de la Red Natura 2000.

\section{Condicionantes abióticos}

A vista de pájaro la estructura del relieve se articula en torno a Puig d'Agulles, punto central y de mayor altitud de Muntayes
d'Ordal. A su alrededor se disponen distintas sierras con direcciones excéntricas, relativamente fáciles de individualizar a causa de la incisión de la red hídrica.

Según Noel Llopis Lladó (1947) la estructura del sector se gesta durante los cambios isostáticos de final del Oligoceno, que marcaron la aparición de grandes fracturas 
longitudinales, con dirección NE-SW, y que posteriormente facilitaron la aparición de fallas de distensión de disposición transversal. Los mismos movimientos tardioligocenos comportaron un basculamiento orogénico hacia el $N E$, que impuso un buzamiento litológico en esta dirección. De esta manera es posible diferenciar cuatro agrupamientos geológicos correspondientes a la era Paleozoica y a los periodos Triásico, Cretácico y Cuaternario.

El basamiento de Muntayes d'Ordal se forma por tres niveles litológicos, entre los que sobresalen por su abundancia pizarras y esquistos del Cambro-Ordoviciano con profusión de diques cuarcíticos. Debido a la morfotectónica estos materiales únicamente afloran en superficie en el sector NE, de mayor buzamiento respecto el plano horizontal (Solé Sabarís et al., 1975). Sobre la serie paleozoica descansa una orla de materiales triásicos, que siguen de manera evidente la disposición NE impuesta por el basamiento. Los materiales se forman por facies germánicas claramente estratificadas de conglomerados y lutitas rojizas del Buntsandstein, donde se encuentran ejemplos de alveolización y taffonización, así como otros ventifactos. Siguen dolomías y calizas del Muschelkalk inferior, lutitas rojizas del Mulschelkalk medio y dolomías, calizas y margas del Muschelkalk superior, así como arcillas y evaporitas del Keuper (Marqués, 1995). Estos últimos materiales del Muschelkalk y del Keuper presentan una gran horizontalidad y configuran relieves en cuesta bien delimitados por los cantiles de su flanco oriental. Estas litologías se encuentran protegidas por una cobertura cretácica que, al seguir el basculamiento general, aparece más desarrollada cuanto más al SW nos situemos. Los materiales predominantes son dolomías del Jurásico y del Cretácico, sobre las cuales se extiende una capa de calizas indiferenciadas y donde resulta fácil encontrar distintas morfologías cársticas, tanto de carácter centimétrico como de medidas superiores (Llopis Lladó, 1947). Los materiales más recientes se localizan de manera profusa en las cercanías de los cursos hídricos, donde se acumulan en terrazas fluviales y en lechos anamostosados; también son muy abundantes los depósitos coluviales, presentes al pie de laderas acusadas y valles encajados.
El volumen y disposición topográfica propician la transición entre una situación climática mediterránea litoral, al sur, a otra ligeramente continentalizada, al norte. Este matiz se demuestra en un ligero incremento de las precipitaciones y un descenso térmico, ligados a una mayor altitud y a particularidades microclimáticas propias de los relieves contrastados, que justifican una inflexión en la isoyeta de los $700 \mathrm{~mm}$ (Clavero, 1994).

\section{Geodiversidad en Muntanyes d'Ordal}

En la Figura $\mathrm{N}^{\circ} 2$ y en el Cuadro $\mathrm{N}^{\circ} 3$ se muestran los resultados generados por el algoritmo utilizado. Los resultados obtenidos señalan unos valores de geodiversidad mayormente medios y altos para el total del área de estudio.

Los valores más discretos se localizan en el sector septentrional y oriental del área de estudio, así como en algunos sectores interiores dominados por materiales cuaternarios y morfologías onduladas. Los valores medios y altos acostumbran a coincidir con ubicaciones montuosas, con un relieve fragmentado, pendientes notorias y un número significativo de elementos abióticos, principalmente derivados de la existencia de morfologías y procesos cársticos. Las calificaciones de geodiversidad muy alta se localizan en topografías similares pero que, frecuentemente, coinciden con litologías triásicas. Esta valoración elevada se explica por los elementos físicos contenidos en el interior de las delimitaciones y, también, por la escasa participación de este tipo de unidades en el conjunto del área de estudio.

El algoritmo empleado asigna valores elevados a aquellas unidades con abundancia de elementos físicos y poca participación de delimitaciones, en número o superficie, en el conjunto. Esta particularidad permite realizar una doble valoración, interna y externa, según se atienda a la geodiversidad del interior de cada unidad delimitada, o a la geodiversidad del conjunto de Muntanyes d'Ordal.

Los valores de geodiversidad baja se concretan en 127 unidades, esto es prácticamente el $31 \%$ de las delimitaciones. Los valores medios son ligeramente superiores, atienden a 145 delimitaciones con una correspon- 
Figura $\mathrm{N}^{\circ} 2$

Geodiversidad en Muntanyes d'Ordal mediante el algoritmo utilizado

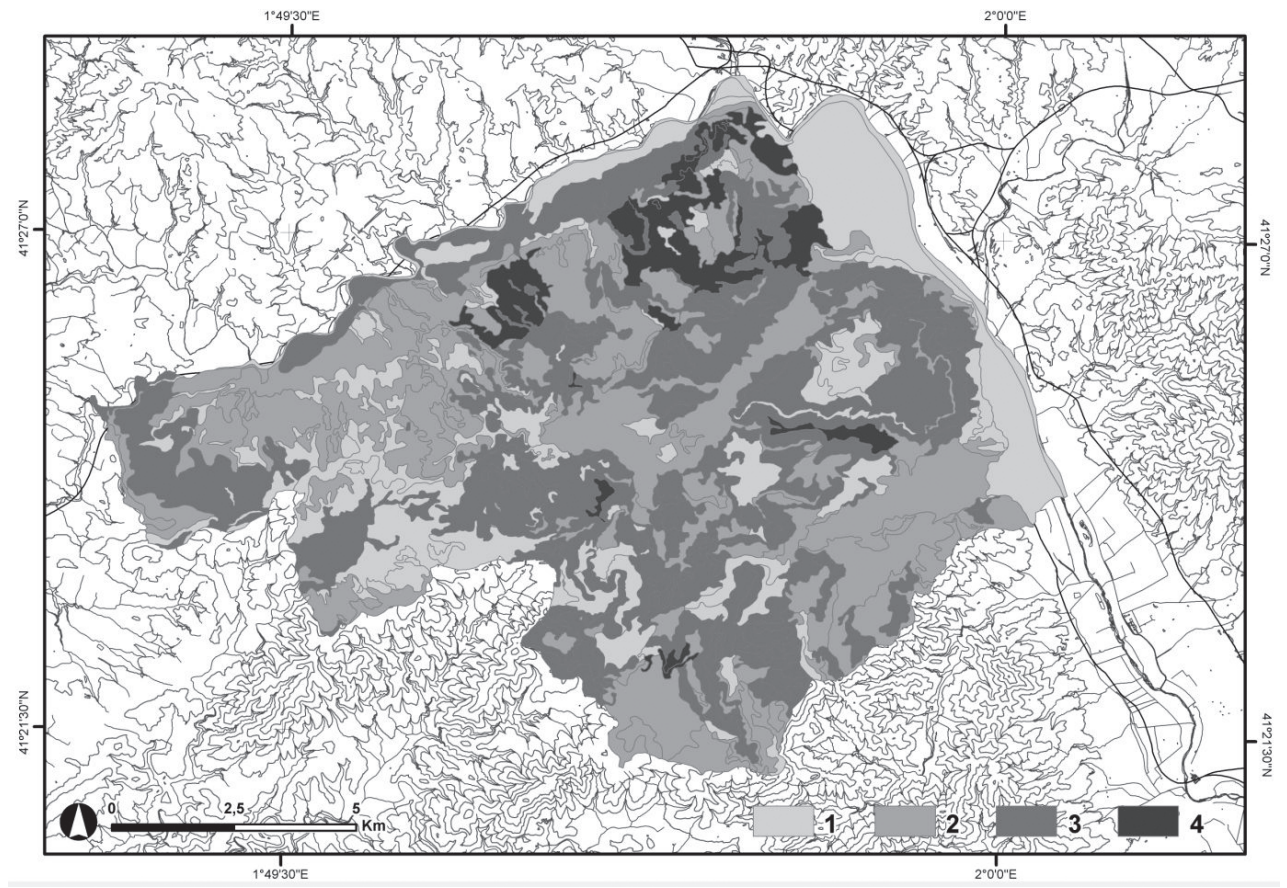

Valores de geodiversidad: 1. bajo; 2. medio; 3.alto; 4. muy alto. Fuente: Elaboración pròpia.

Cuadro $\mathrm{N}^{\circ} 3$

Geodiversidad en Muntanyes d'Ordal

\begin{tabular}{|l|r|r|r|r|}
\hline Geodiversidad & $\begin{array}{c}\text { Número de } \\
\text { unidades }\end{array}$ & $\begin{array}{c}\text { Porcentaje de } \\
\text { unidades }\end{array}$ & $\begin{array}{c}\text { Superficie (ha) } \\
\text { superficie }\end{array}$ \\
\hline Baja & 127 & 30,98 & $3.128,07$ & 20,76 \\
Media & 145 & 35,37 & $5.627,54$ & 37,34 \\
Alta & 122 & 29,76 & $5.462,10$ & 36,24 \\
Muy alta & 16 & 3,9 & 852,64 & 5,66 \\
Total & 410 & 100 & $15.070,35$ & 100 \\
\hline
\end{tabular}

Fuente: Elaboración propia.

dencia de poco más del 35\% del total y una extensión absoluta de prácticamente 5.630 ha. Las unidades con una valoración alta de geodiversidad resultan relativamente numerosas, con una participación que roza el 30\% y 122 compartimentaciones. Finalmente, las unidades de geodiversidad muy alta implican a 16 unidades, con un significado de casi el $4 \%$ del conjunto y una extensión de poco más de 850 ha.

\section{La necesidad de buscar nuevas metodologías para la valoración de la geodiversidad}

El procedimiento utilizado permite estimar la geodiversidad a partir de los elementos que más participan en su definición. Esto es la diversidad y abundancia de elementos físicos, la pendiente y la rugosidad del re- 
lieve. También se considera la participación relativa en número y superficie de cada delimitación realizada, así como la extensión de las unidades observadas y la escala de análisis del estudio. La relación entre las distintas variables se ha establecido a partir de las sugerencias propuestas por otras experiencias (Serrano y Ruiz, 2007a, 2007b; Carcavilla et al., 2007), si bien la particularidad del área de estudio ha requerido modificaciones y ajustes importantes, que propiciaran la obtención de resultados realmente descriptivos de la realidad. Con este fin fue necesario realizar dieciséis iteraciones basadas en un proceso de ensayo y error, en el cual el trabajo de campo y el conocimiento del área de estudio resultaron de vital ayuda. El resultado final se corresponde de forma fidedigna con la realidad y muestra una robustez basada en experiencias previas de probada validez y en un conocimiento del área de estudio de primera mano, que descansa en el trabajo de campo realizado.
El método finalmente desarrollado presenta ciertas divergencias respecto otras experiencias previas. Estas resultan fácilmente observables si se comparan los resultados obtenidos con aquellos resultados ofrecidos por la aplicación de las metodologías originarias. De esta manera, la aplicación del algoritmo explicitado por Serrano y Ruiz (2007a, 2007b) muestra unos valores relativamente cercanos a la realidad con los operadores empleados en el numerador de su formulación (Figura № 3 ), pero que se desvían si se atiende a los utilizados en el denominador (Figura $\mathrm{N}^{\circ} 4$ ). Ello se debe a que este método propone el uso de una corrección logarítmica de los valores de superficie, hecho que permite reducir valores elevados y ajustarlos a escala decimal, para facilitar la comprensión de resultados. Sin embargo, por este mismo motivo, su aplicación en unidades de extensión reducida resulta inapropiada, pues la dimensión de las unidades de referencia llega a comportar la ilógica obtención de resultados con valores negativos.

Figura $\mathrm{N}^{\circ} 3$

Geodiversidad en Muntanyes d'Ordal a través de los operadores empleados en el numerador del algoritmo utilizado

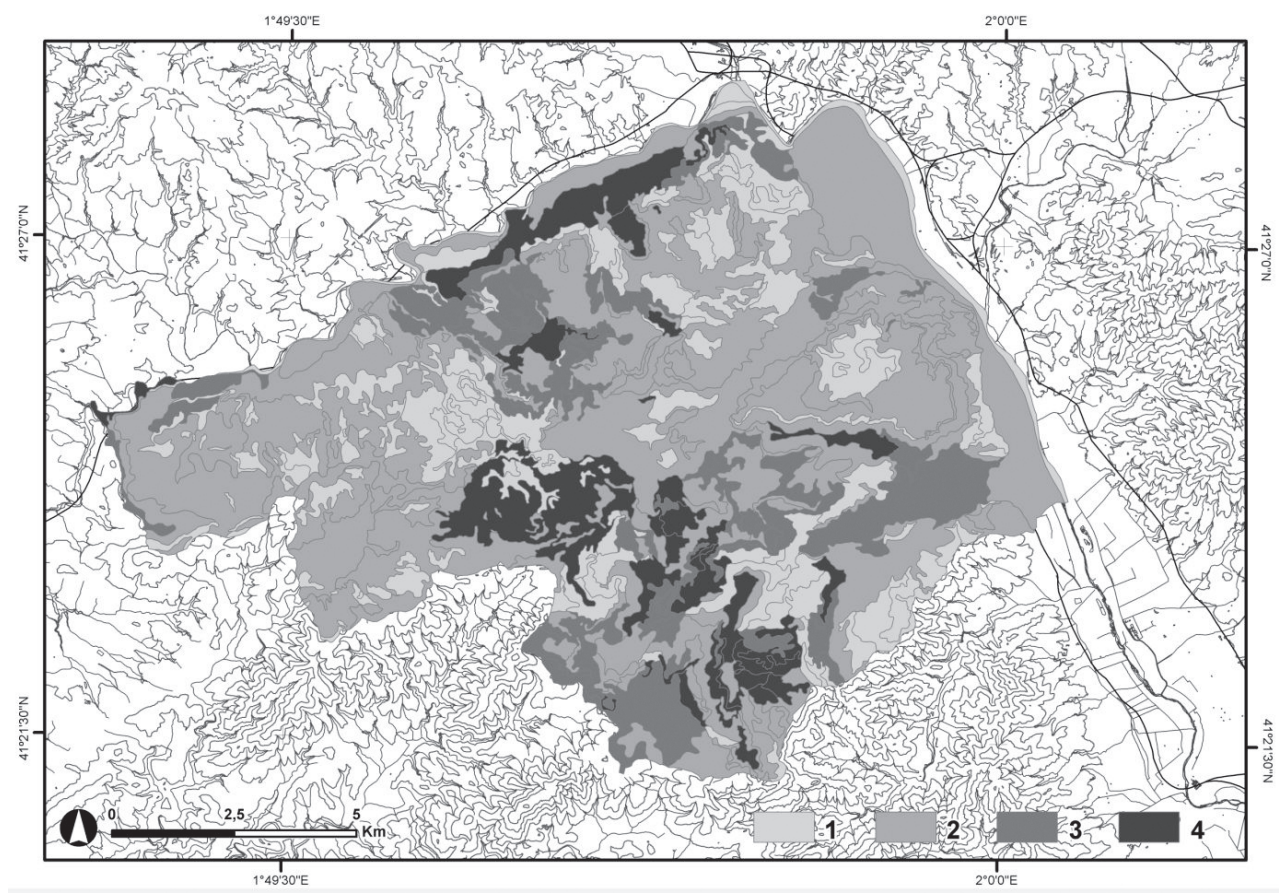

Valores de geodiversidad: 1. bajo; 2. medio; 3. alto; 4. muy alto.

Fuente: elaboración propia a partir de Serrano y Ruiz, 2007a. 
Figura $\mathrm{N}^{\circ} 4$

Geodiversidad en Muntanyes d'Ordal a través de los operadores empleados en el denominador del algoritmo utilizado

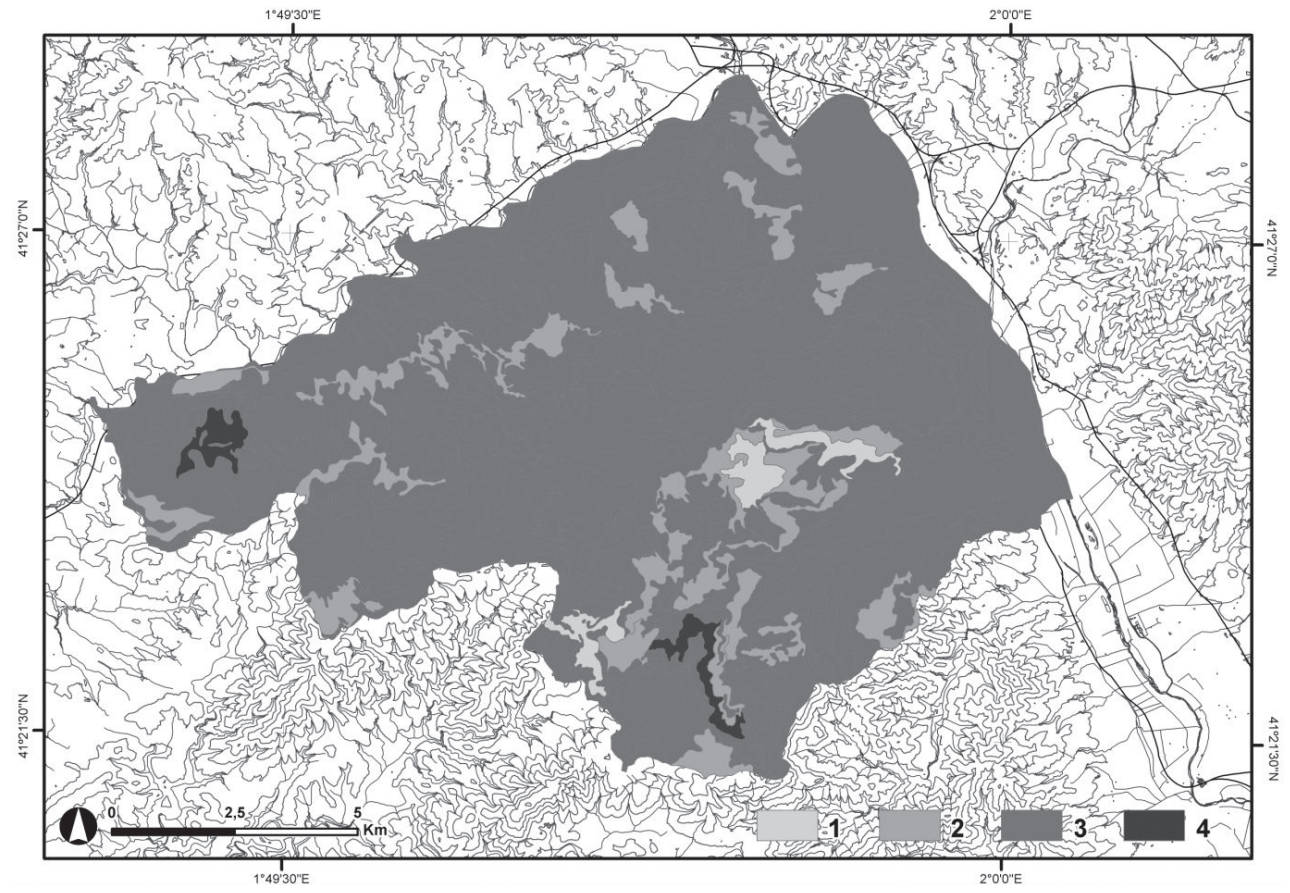

Valores de geodiversidad: 1. bajo; 2. medio; 3. alto; 4. muy alto.

Fuente: Elaboración propia a partir de Serrano y Ruiz, 2007a.

Por otra parte la aplicación de las propuestas de Carcavilla et al. (2007) se ajustan parcialmente a la realidad del área de estudio, particularmente en el cálculo de la geodiversidad intrínseca o abundancia, aunque resulta complicado realizar valoraciones entre distintas unidades (Figura $N^{0} 5$ ), precisamente por las dimensiones del área de estudio y el grado de análisis con el que se realiza su tratamiento. Por otra parte la estimación de la frecuencia de clase que emplean estos autores en su formulación, proporciona unos resultados desviados en nuestro caso, ya que únicamente permite destacar los valores de las unidades de dimensión reducida (Figura $N^{\circ} 6$ ). Este hecho revela la importancia de la dimensión de las áreas de referencia empleadas, precisamente, en función del contexto donde se ubican.

Conviene señalar que trabajos como los de Reynard et al. (2007) o Panizza y Piacente
(2003) evidencian de manera clara el número de variables que deben considerarse a la hora de valorar la geodiversidad, así como la manera de correlacionarlas. Otras experiencias, como las de Calvet y Giusti (2009) destacan la importancia de la escala y la dimensión espacial, en reflexiones que, a partir de la experiencia en Muntanyes d'Ordal, queremos compartir.

La formulación final del algoritmo aplicado se ha establecido tras un largo proceso de ensayo y error. La verificación de los resultados obtenidos se realizó mediante el contraste con la realidad y el conocimiento del área de estudio. Tras dieciséis aproximaciones se obtuvo la formulación aplicada, que es la que mayor coherencia presenta con la realidad y la que más fiabilidad reporta. El algoritmo resulta de cálculo sencillo pues, aunque el número de variables analizadas sea significativo (seis, en total), su interrelación 
Figura $\mathrm{N}^{\circ} 5$

Geodiversidad en Muntanyes d'Ordal (abundancia)

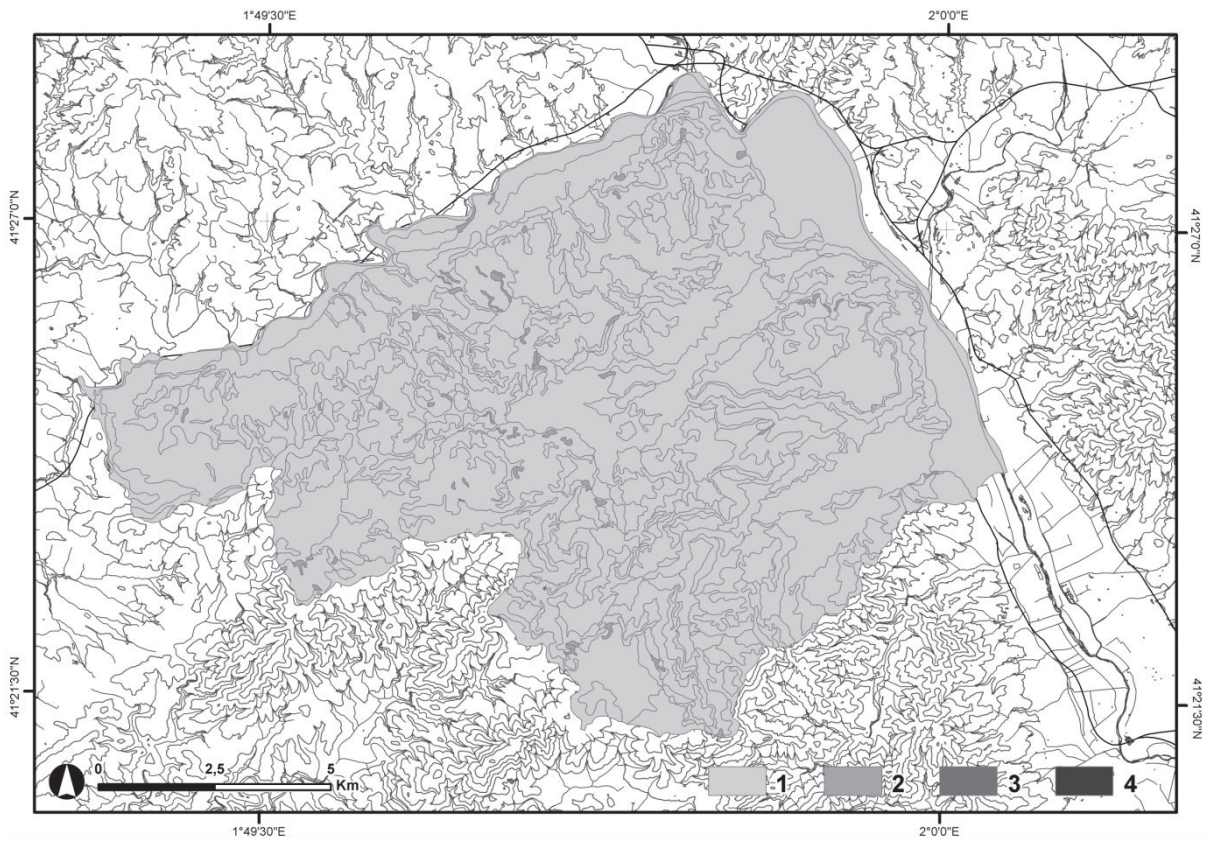

Valores de geodiversidad: 1. bajo; 2. medio; 3. alto; 4. muy alto.

Fuente: Elaboración propia a partir de Carcavilla, López y Durán, 2007.

Figura $\mathrm{N}^{\circ} 6$

Geodiversidad en Muntanyes d'Ordal (frecuencia de clase)

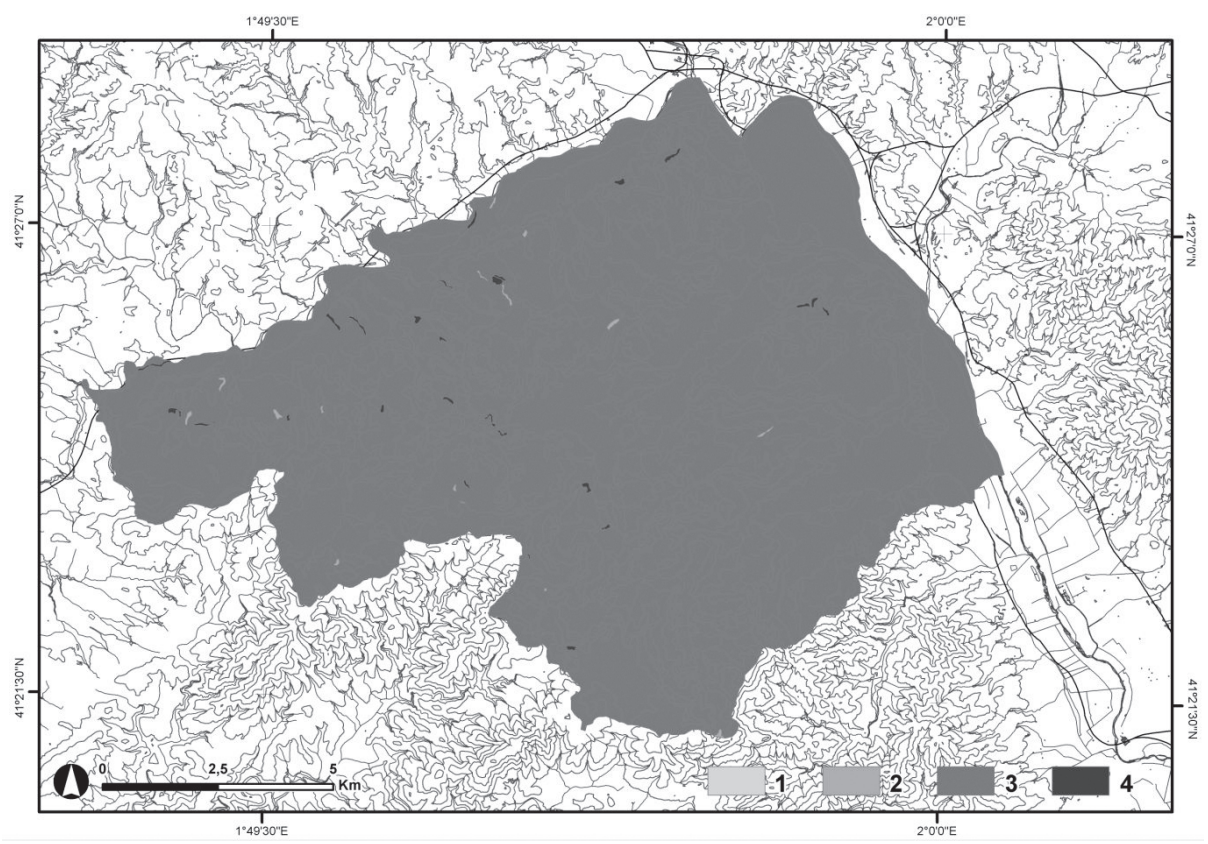

Valores de geodiversidad: 1 . bajo; 2. medio; 3. alto; 4. muy alto.

Fuente: Elaboración propia a partir de Carcavilla, López y Durán, 2007. 
se realiza mediante un álgebra sumamente simple. Durante el proceso de ensayo y error se emplearon algoritmos que consideraban la ponderación de determinadas variables, pero su validez no siempre resultó clara, motivo por el cual terminaron desechándose. También se consideraron cálculos que integraran índices sobre la abundancia relativa de cada clase, como los propuestos por Rosenberg, según recoge Durán (1998). Pero su identificación no siempre pudo realizarse de forma sistemática y su correlación no demostró una validez evidente. De la misma manera se ensayó con procedimientos que homogeneizaban las unidades en función de la participación de estas en el conjunto. Pero los resultados obtenidos no fueron satisfactorios, por otorgar siempre puntuaciones mayores a aquellas delimitaciones más frecuentes o de mayor extensión. A lo largo de todo el proceso metodológico se observó, de forma reiterada, la importancia de ajustar los valores empleados a la realidad impuesta por una escala de trabajo detallada.

La aplicación de este procedimiento en Muntanyes d'Ordal ha revelado un predominio importante de las unidades con una geodiversidad baja y media $(66,35 \%)$, una participación significativa de las unidades con alta geodiversidad $(29,76 \%)$ y una participación minoritaria, pero valiosa de las de muy alta geodiversidad (16 unidades con un significado del $4 \%$ del total). Resulta interesante señalar que las unidades de mayor valoración adquieren este significado, en buena parte, por su carácter singular. Estas tienen una participación en número y en superficie minoritaria en el área de estudio, pero que se encuentra valorada positivamente en el algoritmo utilizado. Esta particularidad permite considerar tanto la geodiversidad interna de cada unidad como la externa, es decir, la del conjunto de Muntanyes d'Ordal. El resultado, en consecuencia, ofrece una doble lectura según si se atiende a la geodiversidad en el interior de cada delimitación o para la totalidad de Muntanyes d'Ordal. Precisamente por este último aspecto conviene recordar que los valores de geodiversidad aquí obtenidos deben ser entendidos en el ámbito donde se insieren y en el contexto escalar en que han sido generados, pues en otros ámbitos se encontrarían descontextualizados.
Finalmente, y como ya se ha notado en otras ocasiones, conviene insistir en la todavía escasa experiencia en valoraciones de geodiversidad y en la aplicación de metodologías no siempre coincidentes que, según la naturaleza del área de estudio, proporcionan resultados con distinto grado de ajuste con la realidad. Un trabajo continuado en este sentido ha de permitir la homogeneización de aproximaciones, particularmente en el caso de los estudios con elevado grado de detalle. Esto es porque, como se ha demostrado, las valoraciones en ámbitos escalares reducidos no muestran el mismo grado de acierto si se aplican en ámbitos escalares grandes. La bondad del método desarrollado ha sido validado, a escala de detalle, en Muntayes d'Ordal. Resultaría positivo que próximas propuestas aplicaran esta misma metodología en otros ámbitos escalares grandes, y que la ajustarán en la medida en que fuera necesario. Dichos próximos ejercicios no solo deberían atender a estas demandas, sino también a otras relacionadas con la valoración cualitativa de la geodiversidad, la estimación de su calidad y la integración con otras medidas y métodos de valoración, tanto de elementos abióticos como de sistemas naturales, en general.

\section{Referencias bibliográficas}

BRUSCHI, V. Desarrollo de una metodología para la caracterización, evaluación y gestión de los recursos de la geodiversidad. Cantabria: Universidad de Cantabria, 2007. Disponible en Internet : http://hdl.handle. net/10803/10611.

CALVET, M. \& GIUSTI, CH. Géosites, géotopes ou géomorphosites ? Pertinence des concepts et complexité scalaire. Géomorphosites, 2009, Vol. 2, p. 101-102. Disponible en Internet: http://geoinfo.amu.edu.pl/iag/ arch/Paris2009Vol2.pdf

CARCAVILLA, L.; LÓPEZ MARTÍNEZ, J. y DURÁN, J.J. Patrimonio geológico y geodiversidad: investigación, conservación, gestión y relación con los espacios naturales protegidos. Madrid: Instituto Geológico y Minero de España, 2007.

CARVAJAL, D.J. y GONZÁLEZ, A. La contribución del patrimonio geológico y minero 
al desarrollo sostenible. Santa Cruz de la Sierra: Coloquio sobre el patrimonio geológicominero en Iberoamérica, 2002.

CASTRO, C. y BRIGNARDELLO, L. Geomorfología aplicada a la ordenación territorial de litorales arenosos. Orientaciones para la protección, usos y aprovechamiento sustentables del sector de Los Choros, comuna de La Higuera, IV Región. Revista de Geografía Norte Grande, 2005, № 33, p. 33-58.

CASTRO, C.; ZÚÑIGA, A. y PATTILLO, C. Geomorfología y geopatrimonio del Mar de Dunas de Atacama, Copiapó $\left(27^{\circ} \mathrm{S}\right)$, Chile. Revista de Geografía Norte Grande, 2012, No 53, p. 123-136.

CHEVAlIER, M. El paisatge de Catalunya. Barcelona: Barcino, 1928.

CLAVERO, P.; RASO, J.M. y MARTÍN, J. Aproximación climatológica para un estudio de paisaje. Análisis climático de Begues. Barcelona: Universidad de Barcelona, 1994.

COMISIÓN EUROPEA. Acción conjunta: un compromiso común para todo el planeta: cooperación internacional en materia de biodiversidad, cambio climático y desertificación. Luxemburgo: Oficina de Publicaciones Oficiales de las Comunidades Europeas, 2006. Disponible en Internet: http://eeas. europa.eu/library/publications/2006_rio_brochure_es.pdf

DELONG, D. Defining biodiversity. Wildlife Society Bulletin, 1996, № 24, p. 738-749.

DURÁN, J.J. El patrimonio geológico de la Comunidad de Madrid. Madrid: Sociedad Geológica de España, 1998.

ERIKSTAD, L. A Holistic approach to secure geoconservation in local physical planning, In: BARETTINO, D.; VALLEJO, M. \& GALLEGO, E. (editors). Towards the Balanced Management and Conservation of the Geological Heritage in the New Millenium. Madrid: Instituto Tecnológico Geominero de España, 1999, p. 69-72.

FERNÁNDEZ, J. y PRADAS, R. Historia de los parques nacionales españoles. La administración conservacionista (1896-2000).
Madrid: Organismo Autónomo de Parques Nacionales, 2002.

GASTON, K. \& SPICER, J. Biodiversity: an introduction. Oxford: Blackwell Science, 2004.

GÓMEZ ORTIZ, A.; OLIVA, M.; SALVÀ, M. y SALVADOR, F. El paisaje como valor patrimonial en los espacios protegidos: el caso del Parque Nacional de Sierra Nevada (España). Scripta Nova. Revista Electrónica de Geografía y Ciencias Sociales, 2010, Vol. XVI, $N^{\circ} 346$. Disponible en Internet: http://www. ub.es/geocrit/sn/sn-346.htm

GONZÁLEZ TRUEBA, J.J. y SERRANO CAÑADAS, E. La valoración del patrimonio geomorfológico en espacios naturales protegidos. Su aplicación al parque nacional de los Picos de Europa. Boletín de la A.G.E., 2008, № 47, p. 147-194.

GRAY, M. Geodiversity. Valuing and conserving abiotic nature. Chichester: Wiley, 2004.

GRONGGRIJP, G. Geodiversity: the key to a holistic approach in renaturation, In: BARETTINO, D.; VAlLejO, M. \& GALlEGO, E. (editors). Towards the Balanced Management and Conservation of the Geological Heritage in the New Millenium. Madrid: Instituto Tecnológico Geominero de España, 1999, p. 77-80.

HAFFEY, D. Local Geodiversity Action plans: A review of progress in England. Sheffield: Nature England Research Report, 2008. Disponible en Internet: http://naturalengland. etraderstores.com/NaturalEnglandShop/Product.aspx?ProductID=812ae460-7b43-408fa195-344023b2fa53

JOHANSSON, C.; ANDERSEN, S. \& ALAPASSI, M., Geodiversity in the Nordic Countries. ProGeo News, 1999, N 1, p. 1-3. Disponible en Internet: http://www.progeo.se/ news/1999/pgn199.pdf

KOZLOWSKI, S. Geodiversity. The concept and scope of geodiversity. Przeglad Geologiczny, 2004, Vol. 52, No 8, p. 833-837. Disponible en Internet: http://www.pgi.gov.pl/ en/wydawnictwa-menu-tekst/link-menu-lewewyd/przeglad-geologiczny/180-przeglpdgeologiczny-2004-082-tom-52 
LLOPIS LLADÓ, N. Contribución al conocimiento de la morfoestructura de los Catalánides. Barcelona: Ariel, 1947.

MALLARACH, J.M. Criteris i mètodes d'avaluació del patrimoni natural. Barcelona: Departament de Medi Ambient, 1999.

MALLARACH, J.M. (coordinador). Protegits, de fet o de dret: primera avaluació del sistema d'espais naturals protegits de Catalunya. Barcelona: Institució Catalana d'Història Natural, 2008.

MARQUÉS, À. La Geologia, En Atles del Baix Llobregat. Barcelona: Institut Cartogràfic de Catalunya, 1995.

NAHARAOUI, F.; EI WARTI, M.; ZAHRAOUI, M. \& SABI, S. Geomorphosite valorization a view to sustainable development: case of Ait Hajji, Oued Boulahmayel Valley, Central Morocco. Journal of Geographic Information System, 2011, No 3, p. 12-17. Disponible en linternet: http://www.scirp.org/ journal/jgis/

NIETO, L.M. Geodiversidad: propuesta de una identificación integradora. Boletín Geológico y Minero, 2001, Vol. 112, No 2, p. 3-12. Disponible en Internet: http://www.igme.es/ internet/Boletin/2001/112_2-2001/1-ARTICULO\%20\%20GEODIVERSIDAD.pdf>.

ONU. Convenio sobre la diversidad biológica. Nueva York: ONU, 1992. Disponible en internet: http://www.cbd.int/doc/legal/cbd-es.pdf

PANIZZA, M. \& PIACENTE, S. Geomorphosites: a bridge between scientific research, cultural integration and artistic suggestion. In: CORATZA, P. \& MARCHETTI, M. (editors). Geomorphological sites: research, assessment and improvement. Modena: Università degli Studi di Modena e Reggio Emilia, 2002, p. 15-20.

PANIZZA, M. \& PIACENTE, S. Geomorfologia culturale. Boloña: Pitagora Editrice, 2003.

PAÜL, V. \& SERRANO, D. Muntanyes d'Ordal. El nom que no surt als mapes. Barcelona: Publicacions de I'Abadia de Montserrat, 2005.
PRALONG, J.P. A method for assessing tourist potencial and use of geomorphological sites. Géomorphologie: relief, processus environnement, 2005, №3, p. 189-196. Disponible en internet: http://geomorphologie.revues. org/index350.html

PRIMACK, R. y ROS, J.D. Introducción a la biología de la conservación. Barcelona: Ariel, 2002.

REYNARD, E. La géomorphologie et la création de paysages. Paysages géomorphologiques, Travaux et recherches, 2004, № 27 , p. 10-18. Disponible en Internet: http://issuu. com/cedocigul/docs/igul-tr27

REYNARD, E. Géotopes, géo(morpho)sites et paysages géomorphologiques. Paysages géomorphologiques, Travaux et recherches, 2004, No 27, p. 123-136. Disponible en internet: http://issuu.com/cedocigul/docs/igul-tr27

REYNARD, E. Paysage et géomorphologie: quelques réflexions sur leurs relations réciproques. In : DROZ, Y.; MIÉVILLE-OTT, V. \& CHÉTELAT, J. (director). La polyphonie du paysage. Lausana: Presses polytechniques et universitaires romandes, 2005, p. 101-124.

REYNARD, E. \& PANIZZA, M. Géomorphosites : définition, évaluation et cartographie. Une introduction. Géomorphologie: relief, processus environnement, 2005, $\mathrm{N}^{\circ} 3$, p. $177-180$.

SERRANO CAÑADAS, E. \& GONZÁLEZ TRUEBA, J. J. Assessment of geomorphosites in protected natural areas: the Picos de Europa National Park (Spain). Geomorphologie, 2005, No 3, p. 197-208. Disponible en Internet: http://geomorphologie.revues.org/ index364.html?file=1

SERRANO CAÑADAS, E. y RUIZ FLAÑO, P. Geodiversidad: concepto, evaluación y aplicación territorial. El caso de Tiermes Caracena (Soria). Boletín de la A.G.E., 2007a, No 45, p. 79-98.

SERRANO CAÑADAS, E. \& RUIZ FLAÑO, P. Geodiversity. Theoretical and applied concept. Geographica Helvetica, 2007b, No 3, p. 140-147. 
SERRANO GINÉ, D. El papel del relieve en la definición de unidades de paisaje. El caso de Muntanyes d'Ordal (Barcelona). Cuadernos de Investigación Geográfica, 2012, Vol. 2, No38, p. 123-145.

SOLÉ SABARÍS, LI. (director). Hoja 420, Hospitalet de Llobregat, Mapa geológico de España 1: 50.000. Madrid: Servicio de Publicaciones del Ministerio de Industria, 1975.

VOTH, A. Los geoparques y el geoturismo: nuevos conceptos de valorización de recursos patrimoniales y desarrollo regional.
En: GALVE, A. (editor). XI Coloquio Ibérico de Geografía. Alcalá de Henares: XI Coloquio Ibérico de Geografía, 2008, p. 1-15.

WIMBLEDON, W.; ISHCHENKO, A.; GERASIMENKO, N.; KARIS, L.; SUOMIEN, V.; JOHANSSON, C. y FREDEN, C. Proyecto geosites, una iniciativa de la Unión Internacional de las ciencias geológicas (IUGS). La ciencia respaldada por la conservación. En: BARETTINO, D.; WIMBLEDON, B. y GALLEGO, E. (editores). Patrimonio geológico: conservación y gestión. Madrid: IGME, 2000, p. 73-100. 